\title{
$n$-Dimensional Fractional Frequency Laplace Transform by the Inverse Difference Operator
}

\author{
M. Meganathan, ${ }^{1}$ Thabet Abdeljawad ${ }^{1},{ }^{2,3,4}$ G. Britto Antony Xavier, ${ }^{1}$ and Fahd Jarad ${ }^{5}$ \\ ${ }^{1}$ Department of Mathematics, Sacred Heart College (Autonomous), Tirupattur District 635601, Vellore, India \\ ${ }^{2}$ Department of Mathematics and General Sciences, Prince Sultan University, P.O. Box 66833, Riyadh 11586, Saudi Arabia \\ ${ }^{3}$ Department of Medical Research, China Medical University, Taichung 40402, Taiwan \\ ${ }^{4}$ Department of Computer Science and Information Engineering, Asia University, Taichung, Taiwan \\ ${ }^{5}$ Department of Mathematics, Cankaya University, Etimesgut 06790, Ankara, Turkey
}

Correspondence should be addressed to Thabet Abdeljawad; tabdeljawad@psu.edu.sa

Received 11 April 2020; Revised 19 June 2020; Accepted 24 July 2020; Published 24 August 2020

Academic Editor: Abdelalim Elsadany

Copyright (c) 2020 M. Meganathan et al. This is an open access article distributed under the Creative Commons Attribution License, which permits unrestricted use, distribution, and reproduction in any medium, provided the original work is properly cited.

With the study of extensive literature on the Laplace transform with one and two variables and its properties, applications are available, but there is no work on $n$-dimensional Laplace transform. In this research article, we define $n$-dimensional fractional frequency Laplace transform with shift values. Several theorems are derived with properties of the Laplace transform. The results are numerically analyzed and discussed through MATLAB.

\section{Introduction}

The fractional calculus is a branch of mathematics that focuses on arbitrary order integrals and derivatives. In spite of that, this type of calculus is as older as the conventional calculus, and it has attracted the interest of researchers for the last few decades. This is because of the results reported by these researchers as consequences of their attempts to model real-world phenomena using the fractional operators [1-4]. The discrete version of these operators fetched the attention of research studies as well. Many good results were reported when fractional sums and differences were used in studying related problems (see [5-17] and the references therein).

The integral transforms such as Mellin, Laplace, and Fourier were applied to obtain the solution of differential equations. These transforms made effectively possible changes of a signal in the time domain into a frequency $s$ domain in the field of digital signal processing (DSP) [18]. The delta Laplace transform was first defined in a very general way by Bohner and Peterson [19]. In 2015, Ivic discussed the discrete Laplace transforms in the view of fast decay factor $e^{-s x}$ and obtained the Laplace transform of
$P(x)$ as $\int_{0}^{\infty} P(x) e^{-s x} \mathrm{~d} x=\pi s^{-2} \sum_{n=1}^{\infty} r(n) e^{-\left(\pi^{2} / n\right)}$. In practice, many applications of Laplace transform (LT), $L[f(x)]=\int_{0}^{\infty} f(x) e^{-s x} \mathrm{~d} x$, and the forward discrete Laplace transform (DLT), $L[f(n)]=\sum_{n=0}^{\infty} f(n) e^{-s n}$, are discussed and mentioned by several authors in [20-23]. For physical applications of Laplace transform, refer [24-27].

In the existing Laplace transform, the shifting value of time domains is one. In 2016, Britto Antony Xavier et al. [28] defined the Laplace transform with shift value $\ell$ using the generalized difference operator and obtained the outcomes of polynomial and trigonometric functions. In this fractional frequency Laplace transform, the shift values $v_{j}^{\prime} s, j=1,2, \ldots, n$ lie in the interval $[0,1]$. In [29], the author introduced the double Laplace transform and applied to solve initial and boundary value problems.

In this research work, we extend the work of Laplace transform into an $n$-dimensional space in discrete case. We present several properties of the fractional transforms for functions such as polynomial factorial, exponential, and trigonometric functions. Also, we derive the relation between Laplace transform and Riemann zeta functions. Furthermore, we present the inverse Laplace transform to 
compare the results with the existing classical Laplace transform for the particular value of $n$.

\section{Preliminaries}

Here, we present some basic definitions and results which will be used further.

Definition 1. Let $u(\bar{t})$ be the function with $n$-variables and $\bar{h} \in R^{n}$ be the shift values. Then, the $n$-dimensional partial difference operator is defined as

$$
\underset{h_{i}}{\Delta} u(\bar{t})=\frac{u\left(t_{1}, t_{2}, \ldots, t_{i}+h_{i}, \ldots, t_{n}\right)-u\left(t_{1}, t_{2}, \ldots, t_{n}\right)}{h_{i}},
$$

where $\bar{t}=\left(t_{1}, t_{2}, \ldots, t_{n}\right)$ and $\bar{h}=\left(h_{1}, h_{2}, \ldots, h_{n}\right)$.

Definition 2 (see [30]). For $h>0$ and $\mu \in R$, the rising $h$-polynomial factorial function is defined as

$$
t_{h}^{[\mu]}=h^{\mu} \frac{\Gamma((t / h)+\mu)}{\Gamma(t / h)},
$$

where $t_{h}^{[0]}=1, \Gamma$ is the Euler gamma function, and $(t / h)+$ $\mu,(t / h) \notin\{0,-1,-2,-3, \ldots\}$ as the division at a pole yields zero.
Lemma 1 (see [31]). Let $h>0$ and $u(t)$ and $w(t)$ be realvalued bounded functions. Then,

$$
\Delta_{h}^{-1}(u(t) w(t))=u(t) \Delta_{h}^{-1} w(t)-\Delta_{h}^{-1}\left(\Delta_{h}^{-1} w(t+h) \Delta_{h} u(t)\right) .
$$

Theorem 1 (see [31]). Let $t \in(0, \infty), h>0$, and $s>0$; then,

$$
L_{h, \nu}\left(t_{h}^{(\mu)}\right)=\frac{h^{\mu+1} \mu ! e^{s^{1 / \nu} h}}{\left(e^{s^{1 / \nu} h}-1\right)^{\mu+1}} .
$$

\subsection{Notations}

(i) $\frac{\Delta}{n(h)} u(\bar{t})=\Delta_{h_{n}} h_{n-1} \cdots \Delta \Delta_{h_{2}} \Delta u\left(t_{1}, t_{2}, \ldots, t_{n}\right)$

(ii) $\Delta_{\frac{-1}{n(h)}}^{-1} u(\bar{t})=\Delta_{h_{n}}^{-1} \Delta_{h_{n-1}}^{-1} \cdots \Delta_{h_{2}}^{-1} \Delta_{h_{1}}^{-1} u\left(t_{1}, t_{2}, \ldots, t_{n}\right)$

(iii) $\mathscr{J}\left(D_{n}\right)$ denotes the set of all subsets of $D_{n}=\{1,2, \ldots, n\}$

(iv) $n\left(D_{n}-\bar{r}\right)$ denotes the number of digits in $D_{n}-\bar{r}$

Definition 3 (infinite inverse principle law). For the function $u(\bar{t})$, we define the infinite inverse principle law as follows:

$$
\left.\Delta \frac{-1}{n(h)}\right|_{\bar{a}} ^{\infty}=\left(\prod_{j=1}^{n} h_{j}\right)\left(\sum_{r_{1}=0}^{\infty} \sum_{r_{2}=0}^{\infty} \ldots \sum_{r_{n}=0}^{\infty} u\left(a_{1}+r_{1} h_{1}, a_{2}+r_{2} h_{2}, \ldots, a_{n}+r_{2} h_{2}\right)\right),
$$

where $\bar{a}=\left(a_{1}, a_{2}, \ldots, a_{n}\right)$. In particular, if $\bar{a}=(0,0, \ldots, 0)$, we obtain

$$
\left.\Delta \frac{-1}{n(h)}\right|_{0} ^{\infty}=\left(\prod_{j=1}^{n} h_{j}\right)\left(\sum_{r_{1}=0}^{\infty} \sum_{r_{2}=0}^{\infty} \cdots \sum_{r_{n}=0}^{\infty} u\left(r_{1} h_{1}, r_{2} h_{2}, \ldots, r_{2} h_{2}\right)\right) .
$$

$$
\Delta \frac{-1}{n(h)} a^{-\sum_{j=1}^{n} s_{j}^{1 / \nu_{j}} t_{j}}=\frac{\left(\prod_{j=1}^{n} h_{j}\right) a^{-\sum_{j=1}^{n} s_{j}^{1 / \nu_{j}} t_{j}}}{\prod_{j=1}^{n}\left(a^{-s_{j}^{1 / v_{j}} h_{j}}-1\right)} .
$$

Proof. Taking $u(\bar{t})=a^{-\sum_{j=1}^{n} s_{j}^{1 / j_{j}} t_{j}}$ in (1) for the shift value $h_{1}$, we obtain

Theorem 2. Let $\bar{t} \in R^{n}, h_{j}>0, j=1,2, \ldots, n$; then,

$$
\underset{h_{1}}{\Delta} a^{-\sum_{j=1}^{n} s_{j}^{1 / v_{j}} t_{j}}=\frac{a^{-\left(s_{1}^{1 / \nu_{1}}\left(t_{1}+h_{1}\right)-\sum_{j=1}^{n} s_{j}^{1 / v_{j}} t_{j}\right)}-a^{-\sum_{j=1}^{n} s_{j}^{1 / v_{j}} t_{j}}}{h_{1}}=\frac{h_{1} a^{-\sum_{j=1}^{n} s_{j}^{1 / v_{j}} t_{j}}}{\left(a^{-s_{1}^{1 / 1 / 1}} h_{1}-1\right)} .
$$

In (8), applying $\Delta_{h_{2}}^{-1}$ on both sides, we get 


$$
\begin{aligned}
& \Delta_{h_{2}}^{-1} \Delta_{h_{1}}^{-1} a^{-\sum_{j=1}^{n} s_{j}^{1 / \nu_{j}} t_{j}}=\frac{h_{1}}{\left(a^{-s_{1}^{1 / \nu_{1}} h_{1}}-1\right)} \Delta_{h_{2}}^{-1} a^{-\sum_{j=1}^{n} s_{j}^{1 / v_{j}} t_{j}}
\end{aligned}
$$

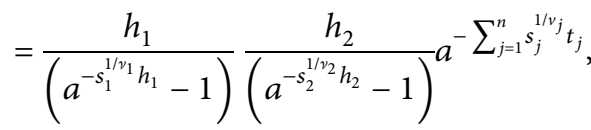

$$
\begin{aligned}
& \Delta_{h_{2}}^{-1} \Delta_{h_{1}}^{-1} a^{-\sum_{j=1}^{n} s_{j}^{1 / v_{j}} t_{j}}=\frac{\left(\prod_{j=1}^{2} h_{j}\right) a^{-\sum_{j=1}^{n} s_{j}^{1 / v_{j}} t_{j}}}{\prod_{j=1}^{2}\left(a^{-s_{j}^{1 / v_{j}} h_{j}}-1\right)} .
\end{aligned}
$$

Proceeding like this for the induction on $n$, we get the proof of (7).

Corollary 1. Let $\bar{t} \in R^{n}, h_{j}>0$ and $j=1,2, \ldots, n$. Then, we have

$$
\Delta_{n(h)}^{-1} e^{-\sum_{j=1}^{n} s_{j}^{1 / v_{j}} t_{j}}=\frac{\left(\prod_{j=1}^{n} h_{j}\right) e^{-\sum_{j=1}^{n} s_{j}^{1 / v_{j}} t_{j}}}{\prod_{j=1}^{n}\left(e^{-s_{j}^{1 / v_{j}} h_{j}}-1\right)} .
$$

Proof. In the proof of Theorem 2, replacing $a$ by $e$, we get (10).

Corollary 2. In Theorem 2, applying $a=3$, we get

$$
\Delta \frac{-1}{n(h)} 3^{-\sum_{j=1}^{n} s_{j}^{1 / v_{j}} t_{j}}=\frac{\left(\prod_{j=1}^{n} h_{j}\right) 3^{-\sum_{j=1}^{n} s_{j}^{1 / v_{j}} t_{j}}}{\prod_{j=1}^{n}\left(3^{-s_{j}^{1 / v_{j}} h_{j}}-1\right)} .
$$

Example 1. Let $n=2$ in (11); we get the result for the shift values $h_{1}$ and $h_{2}$ as

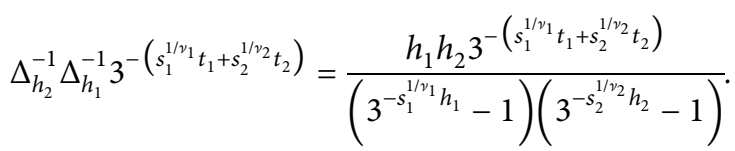

Summing from 0 to $\infty$ for $t_{1}$ and $t_{2}$ on both sides yields

$$
\left.\left.\Delta_{h_{2}}^{-1} \Delta_{h_{1}}^{-1} 3^{-\left(s_{1}^{1 / v_{1}} t_{1}+s_{2}^{1 / \nu_{2}} t_{2}\right)}\right|_{0} ^{\infty}\right|_{0} ^{\infty}=\frac{h_{1} h_{2}}{\left(3^{-s_{1}^{1 / 1 / 1}} h_{1}-1\right)\left(3^{-s_{2}^{1 / v_{2}} h_{2}}-1\right)} \text {. }
$$

For $n=2$, the infinite principle law reads

$$
\left.\left.\Delta_{h_{2}}^{-1} \Delta_{h_{1}}^{-1} u\left(t_{1}, t_{2}\right)\right|_{0} ^{\infty}\right|_{0} ^{\infty}=h_{1} h_{2} \sum_{r_{1}=0}^{\infty} \sum_{r_{2}=0}^{\infty} u\left(r_{1} h_{1}, r_{2} h_{2}\right) \text {. }
$$

Equating (13) and (14) for the function $u\left(t_{1}, t_{2}\right)=3^{-\left(s_{1}^{1 / 1 / 1} t_{1}+s_{2}^{1 / \nu_{2}} t_{2}\right)}$, we obtain

$$
h_{1} h_{2} \sum_{r_{1}=0}^{\infty} \sum_{r_{2}=0}^{\infty} 3^{-\left(s_{1}^{1 / 1 / 1} r_{1} h_{1}+s_{2}^{1 / 2 / r_{2}} r_{2} h_{2}\right)}=\frac{h_{1} h_{2}}{\left(3^{-s_{1}^{1 / 1 / 1}} h_{1}-1\right)\left(3^{-s_{2}^{1 / 2 / 2} h_{2}}-1\right)} \text {, }
$$

which is verified for the particular values $s_{1}=2, s_{2}=3, v_{1}=0.3, v_{2}=0.5, h_{1}=0.4$, and $h_{2}=0.7$ by MATLAB coding as follows: $((0.4) . *(0.7)) . *$ $\operatorname{symsum}(\operatorname{symsum}(3 . \wedge(-\quad(2 . \wedge(1 . / 0.3) . * 0.4 . * r 1+3 . \wedge(1 . /$ $0.5) . * 0.7 . * r 2)), r 1,0, \infty), r 2,0, \infty)=(0.4 . * 0.7) /((3 . \wedge(-$ $(2 . \wedge(1 . / \quad 0.3) . * 0.4))-1) . *(3 . \wedge(-(3 . \wedge(1 . / 0.5) . \quad * 0.7))-$ 1)) $=0.2837$.

\section{3. n-Dimensional Fractional Frequency Laplace Transform}

Definition 4. For the function $u(\bar{t})$ with $n$-variables $t_{1}, t_{2}, \ldots, t_{n}$, the $n$-dimensional fractional frequency Laplace transform is defined as

$$
\begin{aligned}
\mathscr{L}_{n(h)}[u(\bar{t})] & =U_{n}(\bar{s})=\left.\Delta \frac{-1}{n(h)} u(\bar{t}) e^{-\sum_{j=1}^{n} s_{j}^{1 / v_{j}} t_{j}}\right|_{t_{j}=0, j=1,2, \ldots, n} ^{\infty} \\
& =\left(\prod_{j=1}^{n} h_{j}\right) \sum_{r_{1}=0}^{\infty} \sum_{r_{2}=0}^{\infty} \cdots \sum_{r_{n}=0}^{\infty} u\left(r_{1} h_{1}, r_{2} h_{2}, \ldots, r_{n} h_{n}\right) e^{-\sum_{j=1}^{n} s_{j}^{1 / \nu_{j}} r_{j} h_{j}} .
\end{aligned}
$$

Remark 1

(i) The $n$-dimensional fractional frequency Laplace transform satisfies the linear property.

(ii) In the aforesaid equation (16), we represent the Laplace transform of the functions in two ways: one in the closed-form solution and another one in the summation form solution. In this paper, we numerically verified and analyzed with MATLAB that both solutions are equal.
Theorem 3. Let $\bar{t} \in R^{n}, \bar{h}>0, \quad v_{j}$ be a fraction, and $s_{j}>0, j=1,2, \ldots, n$. Then, we have

$$
\mathscr{L}_{n(h)}[1]=\frac{\prod_{j=1}^{n} h_{j}}{\prod_{j=1}^{n}\left(e^{-s_{j}^{1 / j_{j}} h_{j}}-1\right)} .
$$

Proof. Taking $u(\bar{t})=1$ in (16) yields 


$$
\begin{aligned}
& \mathscr{L}_{n(h)}[1]=\left.\Delta_{n(h)}^{-1} e^{-\sum \sum_{j=1}^{n} s_{j}^{1 / v_{j}} t_{j}}\right|_{t_{j}=0, j=1,2, \ldots, n} ^{\infty} \\
& =\left.\left.\left.\Delta_{h_{n}}^{-1} e^{-s_{n}^{1 / v_{n}} t_{n}}\right|_{0} ^{\infty} \cdots \Delta_{h_{2}}^{-1} e^{-s_{2}^{1 / \nu_{2}} t_{2}}\right|_{0} ^{\infty} \Delta_{h_{1}}^{-1} e^{-s_{1}^{1 / v_{1}} t_{1}}\right|_{0} ^{\infty} \\
& =\frac{h_{n}}{\left(e^{-s_{n}^{1 / v_{n}} h_{n}}-1\right)} \cdots \frac{h_{2}}{\left(e^{-s_{2}^{1 / \nu_{2}} h_{2}}-1\right)} \frac{h_{1}}{\left(e^{-s_{1}^{1 / \nu_{1}} h_{1}}-1\right)},
\end{aligned}
$$

(using Corollary 1), which completes the proof.

Theorem 4. Let $\bar{t} \in R^{n}, \bar{h}>0, \quad v_{j}$ be a fraction, and $s_{j}>0, j=1,2, \ldots, n$; then,

$$
\mathscr{L}_{n(h)}\left[e^{\sum_{j=1}^{n} a_{j} t_{j}}\right]=\frac{\prod_{j=1}^{n} h_{j}}{\prod_{j=1}^{n}\left(e^{-\left(s_{j}^{1 / v_{j}}-a_{j}\right) h_{j}}-1\right)} .
$$

Proof. Taking $u(\bar{t})=e^{\sum_{j=1}^{n} a_{j} t_{j}}$ in (16), we have

$$
\begin{aligned}
\mathscr{L}_{n(h)}\left[e^{\sum_{j=1}^{n} a_{j} t_{j}}\right] & =\left.\Delta_{n(h)}^{-1} e^{\sum_{j=1}^{n} a_{j} t_{j}} e^{-\sum_{j=1}^{n} s_{j}^{1 / v_{j}} t_{j}}\right|_{t_{j}=0, j=1,2, \ldots, n} ^{\infty} \\
& =\left.\left.\left.\Delta_{h_{n}}^{-1} e^{-\left(s_{n}^{1 / v_{n}}-a_{n}\right) t_{n}}\right|_{0} ^{\infty} \cdots \Delta_{h_{2}}^{-1} e^{-\left(s_{2}^{1 / v_{2}}-a_{2}\right) t_{2}}\right|_{0} ^{\infty} \Delta_{h_{1}}^{-1} e^{-\left(s_{1}^{1 / v_{1}}-a_{1}\right) t_{1}}\right|_{0} ^{\infty} \\
& =\frac{h_{n}}{\left(e^{-\left(s_{n}^{1 / v_{n}}-a_{n}\right) h_{n}}-1\right)} \cdots \frac{h_{2}}{\left(e^{-\left(s_{2}^{1 / v_{2}}-a_{2}\right) h_{2}}-1\right)} \frac{h_{1}}{\left(e^{-\left(s_{1}^{1 / v_{1}}-a_{1}\right) h_{1}}-1\right)},
\end{aligned}
$$

which gives (19).

Example 2. For $n=2$, the summation solution of the exponential function given by the infinite inverse principle law and the closed form of the solution given as

$$
\begin{aligned}
\mathscr{L}_{n(h)}\left[e^{a_{1} t_{1}+a_{2} t_{2}}\right] & =h_{1} h_{2} \sum_{r_{2}=0}^{\infty} \sum_{r_{1}=0}^{\infty} e^{-\left[\left(s_{1}^{1 / \nu_{1}}-a_{1}\right) r_{1} h_{1}+\left(s_{2}^{1 / \nu_{2}}-a_{2}\right) r_{2} h_{2}\right]} \\
& =\frac{h_{2}}{\left(e^{-\left(s_{2}^{1 / \nu_{2}}-a_{2}\right) h_{2}}-1\right)} \frac{\left.h_{1}\right)}{\left(e^{-\left(s_{1}^{1 / \nu_{1}}-a_{1}\right) h_{1}}-1\right)}
\end{aligned}
$$

is numerically verified for the particular values $h_{1}=7, h_{2}=3, a_{1}=5, a_{2}=9, v_{1}=0.1, v_{2}=0.3, s_{1}=11$, and $s_{2}=13$ by MATLAB coding as follows: 21. * (symsum (symsum $(\exp (-(11 . \wedge(1 . / 1.0)-5) . * 7 . * r 1$ $+(13 . \wedge(1 . / 0.3)-9 . * 3 . * r 2)), \quad r 1,0,10), r 2,0,10))=21 . /$ $((\exp (-(11 . \wedge(1 . / 0.1)-5) . * 7)-1) . *(\exp (-(13 . \wedge(1 . / 0.3)$ $-9) . * 3)-1))$.

The following are the graphical representations of the exponential function in time and frequency domains. Figure 1 is the graphical representation of the input function $e^{5 t_{1}+9 t_{2}}$ in the time domain, and Figure 2 is the graphical representation of the output in the frequency domain for the particular values of $v_{1}=0.0001, v_{2}=0.0003$. One can easily choose the values of fraction $v_{i}^{\prime} s$ to get the output in the frequency domain.
Theorem 5. Let $\bar{t} \in R^{n}, \bar{h}>0, \quad v_{j}$ be a fraction, and $s_{j}>0, j=1,2, \ldots, n$; then,

$$
\mathscr{L}_{n(h)}\left[e^{i \sum_{j=1}^{n} a_{j} t_{j}}\right]=\frac{\prod_{j=1}^{n} h_{j} \sum_{\bar{r} \in \mathcal{F}\left(D_{n}\right)}(-1)^{n\left(D_{n}-\bar{r}\right)} e_{-i\left(a_{\bar{r}} h_{\bar{r}}\right)} e_{s_{D_{n}-\bar{r}} h_{D_{n}-\bar{r}}}}{2^{n}\left[\prod_{j=1}^{n}\left(\cosh s_{j}^{1 / v_{j}} h_{j}-\cos a_{j} h_{j}\right)\right]}
$$

where $e_{-i\left(a_{\bar{r}} h_{\bar{r}}\right)}=e^{-i\left(a_{1} h_{1}+a_{2} h_{2}+\cdots+a_{n} h_{n}\right)}$ for $\bar{r}=\{1,2, \ldots, n\}$,

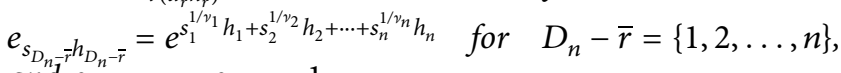
and $e_{-i\left(a_{0} h_{0}\right)}=e_{s_{0} h_{0}}=1$.

Proof. From the previous theorem, we obtain the Laplace transform for the trigonometric function $e^{i \sum_{j=1}^{n} a_{j} t_{j}}$ as

$$
\mathscr{L}_{n(h)}\left[e^{i \sum_{j=1}^{n} a_{j} t_{j}}\right]=\frac{\prod_{j=1}^{n} h_{j}}{\prod_{j=1}^{n}\left(e^{-\left(s_{j}^{1 / v_{j}}-i a_{j}\right) h_{j}}-1\right)} .
$$

The proof then can be continued by making use of the conjugate and the product of each term in $n$-variables.

Theorem 6. Let $\bar{t} \in R^{n}, \bar{h}>0, \quad v_{j}$ be a fraction, and $s_{j}>0, j=1,2, \ldots, n$; then, 


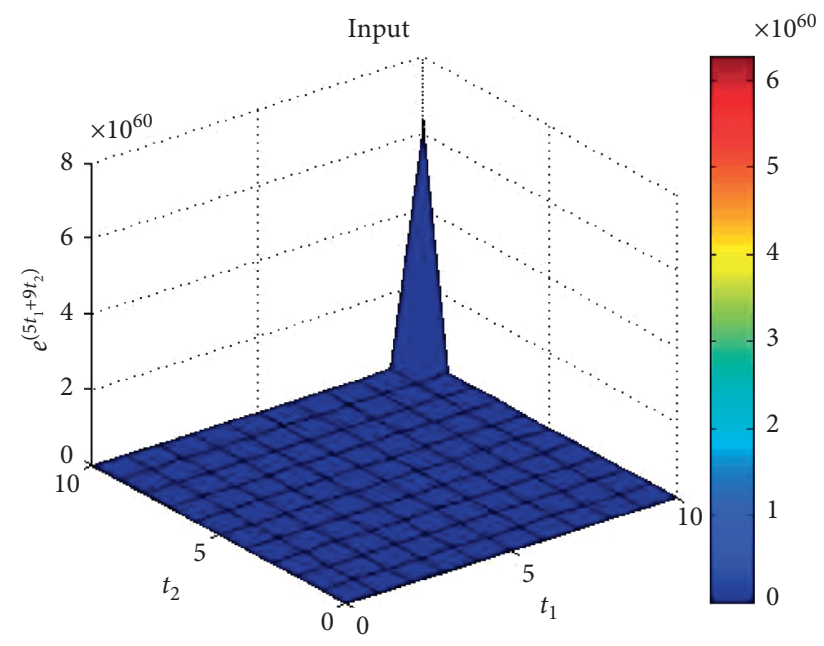

Figure 1: Time signal (function) $e^{5 t_{1}+9 t_{2}}$.

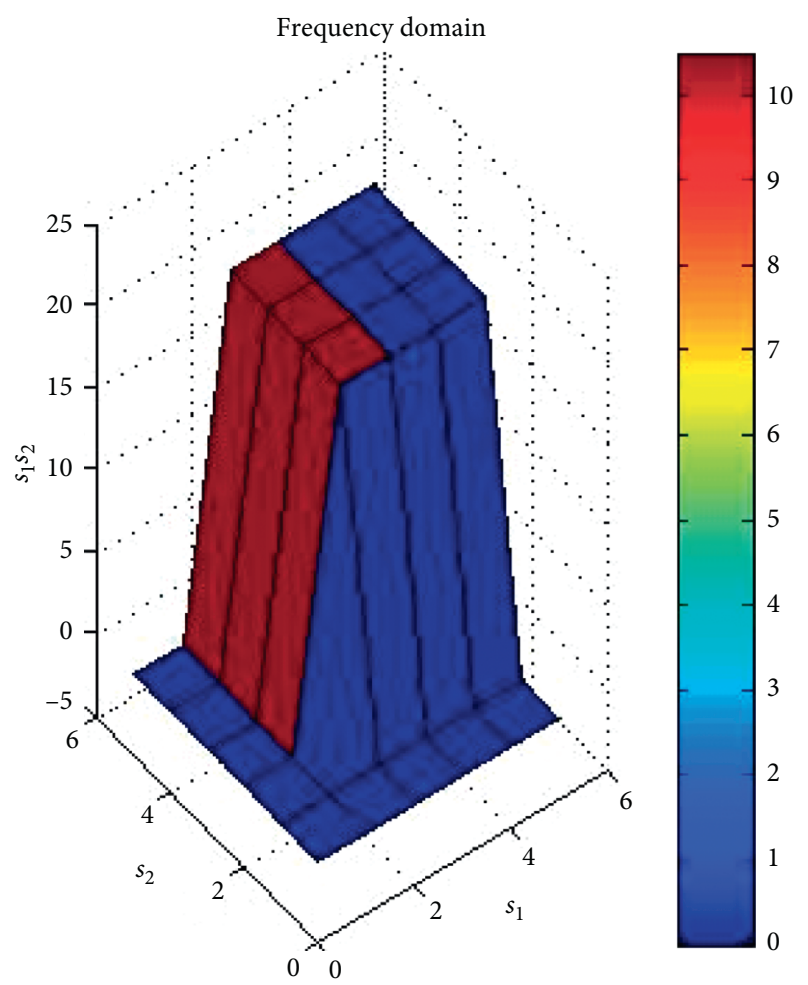

$v_{1}=0.001, v_{2}=0.0003$

FIGURE 2: Frequency signal for $v_{1}=0.0001$ and $v_{2}=0.0003$. 


$$
\begin{gathered}
\mathscr{L}_{2(h)}\left[\sin \left(\sum_{j=1}^{2} a_{j} t_{j}\right)\right]=\frac{h_{1} h_{2}\left(e^{s_{2}^{1 / v_{2}} h_{2}} \sin a_{1} h_{1}+e^{s_{1}^{1 / \nu_{1}} h_{1}} \sin a_{2} h_{2}-\sin \left(a_{1} h_{1}+a_{2} h_{2}\right)\right)}{4\left(\prod_{j=1}^{2}\left(\cosh s_{j}^{1 / v_{j}} h_{j}-\cos a_{j} h_{j}\right)\right)}, \\
\mathscr{L}_{2(h)}\left[\cos \left(\sum_{j=1}^{2} a_{j} t_{j}\right)\right]=\frac{h_{1} h_{2}\left(\cos \left(\sum_{j=1}^{2} a_{j} t_{j}\right)-e^{s_{2}^{1 / v_{2}} h_{2}} \cos a_{1} h_{1}-e^{s_{1}^{1 / v_{1}} h_{1}} \cos a_{2} h_{2}+e^{\sum_{j=1}^{2} s_{j}^{1 / v_{j}} h_{j}}\right)}{4\left(\prod_{j=1}^{2}\left(\cosh s_{j}^{1 / v_{j}} h_{j}-\cos a_{j} h_{j}\right)\right)} .
\end{gathered}
$$

Proof. The proof follows by taking $n=2$ in Theorem 5, making the product by its conjugate terms and separating the real and imaginary parts to get the double Laplace transform for the sine and cosine terms.
Example 3. Equation (24) is the closed-form solution of the sine function. Now, for $n=2$, the summation solution of the sine function given by the infinite inverse principle law is

$$
\mathscr{L}_{2(h)}\left[\sin \left(\sum_{j=1}^{2} a_{j} t_{j}\right)\right]=h_{1} h_{2} \sum_{r_{2}=0}^{\infty} \sum_{r_{1}=0}^{\infty} \sin \left(a_{1} r_{1} h_{1}+a_{2} r_{2} h_{2}\right) e^{-\left(s_{1}^{1 / \nu_{1}} r_{1} h_{1}+s_{2}^{1 / \nu_{2}} r_{2} h_{2}\right)} .
$$

Now, (24) and (26) are numerically verified for the particular values $h_{1}=2, h_{2}=3, a_{1}=1, a_{2}=4, v_{1}=0.4, v_{2}=$ $0.6, s_{1}=5$, and $s_{2}=6$ by MATLAB coding as follows: 6. * symsum (symsum $(\sin (2 . * r 1+12 . * r 2) . * \exp (-(5 . \wedge$ $(1 . / 0.4) . * 2 . * r 1+6 . \wedge(1 . / 0.6) . * 3 . * r 2)), r 1,0,10), \quad r 2,0$, $\infty)=(6 . *(\exp (6 . \wedge(1 . / 0.6) . * 3) . * \sin (2)-\exp ((5 . \wedge(1 . /$ $0.4) . * 2) . * \sin (12)-\sin (14))) /(4 . *(\cosh (6 . \wedge(1 . / 0.6) . *$ $3)-\cos (12)) . *((\cosh (5 . \wedge(1 . / 0.4)) . * 2)-\cos (2))))$.

The following are the graphical representations of the sine function in time and frequency domains. Figure 3 represents the input time-domain signal (function) for the sine function. Figure 4 represents the output in the frequency domain for the particular values of $v_{1}=0.4$ and $v_{2}=0.6$. Figure 5 represents the output in the frequency domain for the particular values of $\nu_{1}=0.3$ and $\nu_{2}=0.5$. Figure 6 represents the output in the frequency domain for the particular values of $v_{1}=0.1$ and $v_{2}=0.7$. Similarly, one can analyze the solution in the frequency domain by choosing diverse values of fraction $v_{i}^{\prime} s$.

Theorem 7. Let $\bar{t} \in R^{n}, \bar{h}>0, \quad v_{j}$ be a fraction, and $s_{j}, \mu_{j}>0, j=1,2, \ldots, n$; then,

$$
\mathscr{L}_{n(h)}\left[\prod_{j=1}^{n}\left(t_{j}\right)_{h_{j}}^{\left(\mu_{j}\right)}\right]=\prod_{j=1}^{n} \frac{h_{j}^{\mu_{j}+1} \mu_{j} ! e^{s_{j}^{1 / v_{j}} h_{j}}}{\left(e^{-s_{j}^{1 / v_{j}}}-1\right)} .
$$

Proof. Taking $u(\bar{t})=\prod_{j=1}^{n}\left(t_{j}\right)_{h_{j}}^{\left(\mu_{j}\right)}$ in (16), we have

$$
\begin{aligned}
& \mathscr{L}_{n(h)}\left[\prod_{j=1}^{n}\left(t_{j}\right)_{h_{j}}^{\left(\mu_{j}\right)}\right]=\left.\Delta \frac{-1}{n(h)} \prod_{j=1}^{n}\left(t_{j}\right)_{h_{j}}^{\left(\mu_{j}\right)} e^{-\sum_{j=1}^{n} s_{j}^{1 / v_{j}} t_{j}}\right|_{t_{j}=0, j=1,2, \ldots, n} ^{\infty} \\
& =\left.\left.\left.\Delta_{h_{n}}^{-1}\left(t_{n}\right)_{h_{n}}^{\left(\mu_{n}\right)} e^{-s_{n}^{1 / \nu_{n}} t_{n}}\right|_{0} ^{\infty} \cdots \Delta_{h_{2}}^{-1}\left(t_{2}\right)_{h_{2}}^{\left(\mu_{2}\right)} e^{-s_{2}^{1 / \nu_{2}} t_{2}}\right|_{0} ^{\infty} \Delta_{h_{1}}^{-1}\left(t_{1}\right)_{h_{1}}^{\left(\mu_{1}\right)} e^{-s_{1}^{1 / \nu_{1}} t_{1}}\right|_{0} ^{\infty}
\end{aligned}
$$

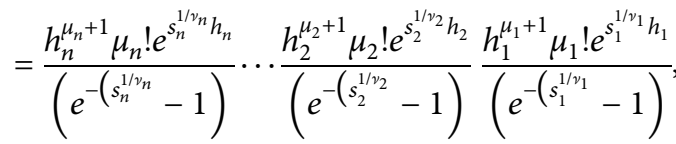




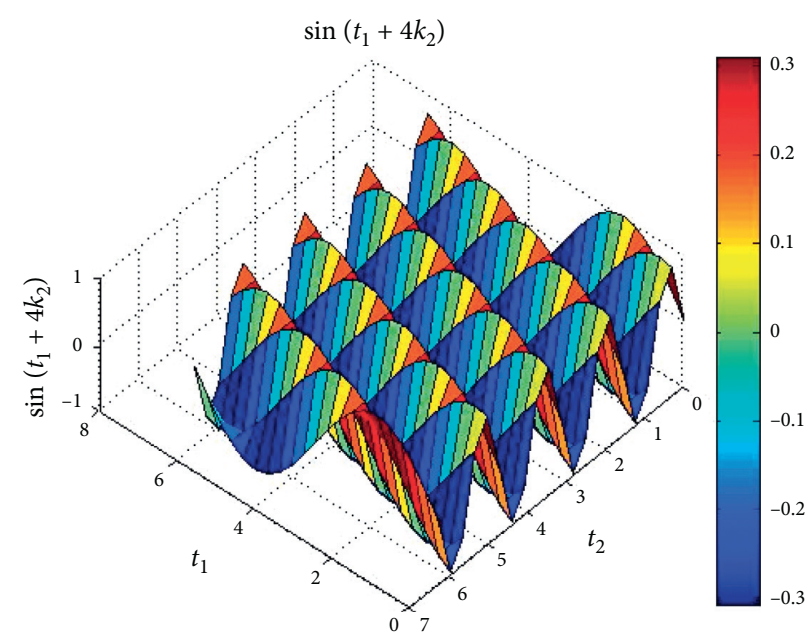

Figure 3: Time signal for $\sin \left(t_{1}+4 t_{2}\right)$.

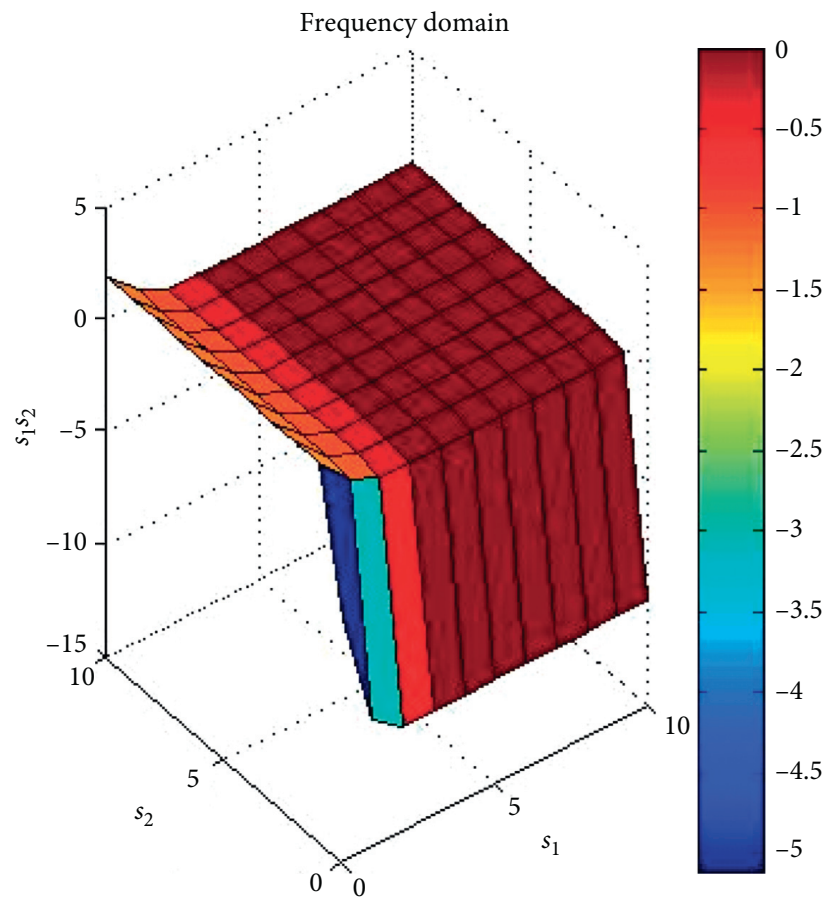

$v_{1}=0.4, v_{2}=0.6$

FIGURE 4: Frequency signal for $v_{1}=0.4$ and $v_{2}=0.6$.

by Lemma 1, which gives (4).

$$
\zeta(\mu)=\sum_{s=1}^{\infty} \frac{1}{s^{\mu}}
$$

3.1. n-Kind Riemann Zeta Function in the Discrete Case. In Theorem 7, when $v_{j}=1$ and $h_{j} \longrightarrow 0$ for $j=1,2, \ldots, n$., we get

$$
\mathscr{L}_{n(h)}\left[t_{1}^{\mu_{1}-1} t_{2}^{\mu_{2}-1} \ldots t_{n}^{\mu_{n}-1}\right]=\frac{\Gamma\left(\mu_{n}\right) \ldots \Gamma\left(\mu_{2}\right) \Gamma\left(\mu_{1}\right)}{s_{n}^{\mu_{n}} \ldots s_{2}^{\mu_{2}} s_{1}^{\mu_{1}}} .
$$

Equation (29) can be written as

$$
\frac{\Gamma\left(\mu_{n}\right) \ldots \Gamma\left(\mu_{2}\right) \Gamma\left(\mu_{1}\right)}{s_{n}^{\mu_{n}} \ldots s_{2}^{\mu_{2}} s_{1}^{\mu_{1}}}=\left.\left.\Delta_{h_{n}}^{-1} t_{n}^{\mu_{n}-1} e^{-s_{n} t_{n}}\right|_{0} ^{\infty} \cdots \Delta_{h_{2}}^{-1} t_{2}^{\mu_{2}-1} e^{-s_{2} t_{2}}\right|_{0} ^{\infty}
$$$$
\left.\cdot \Delta_{h_{1}}^{-1} t_{1}^{\mu_{1}-1} e^{-s_{1} t_{1}}\right|_{0} ^{\infty}
$$

We know that the Riemann zeta function is defined as 


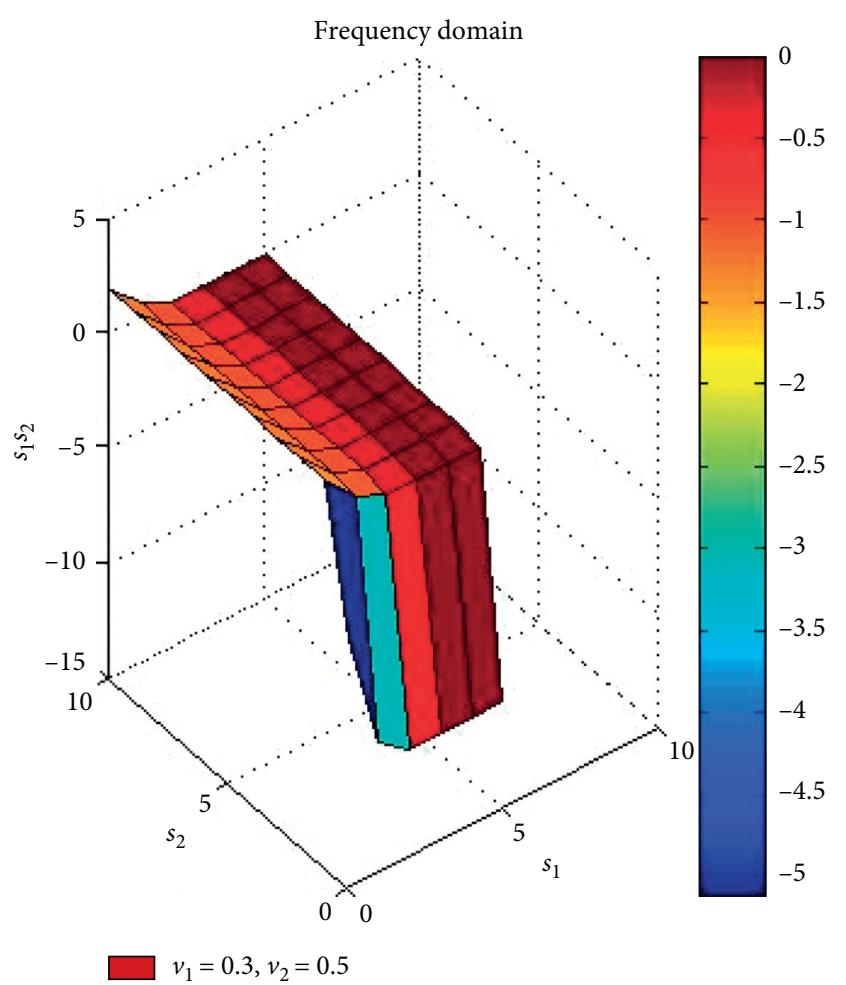

FIgURE 5: Frequency signal for $v_{1}=0.3$ and $v_{2}=0.5$.

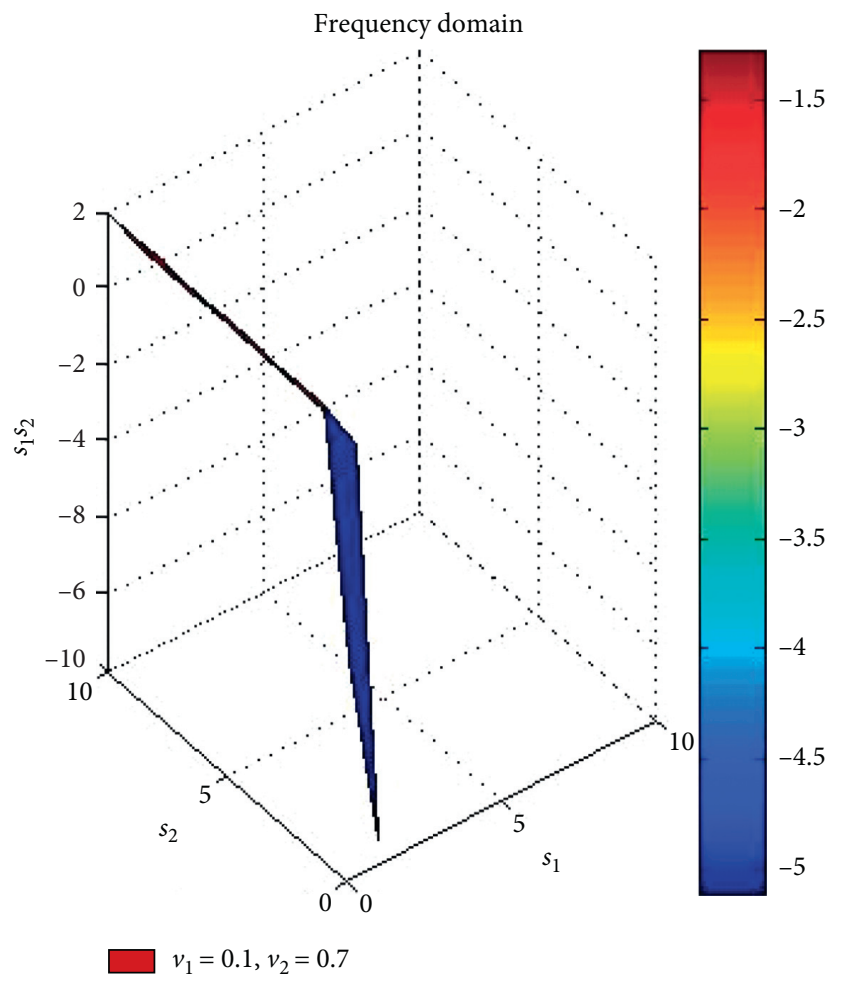

FIGURE 6: Frequency signal for $v_{1}=0.1$ and $v_{2}=0.7$. 
Taking summation on $s_{j}, j=1,2, \ldots, \infty$, on both sides, we get

$$
\begin{gathered}
\prod_{j=1}^{n} \Gamma\left(\mu_{j}\right) \sum_{s_{n}=1}^{\infty} \frac{1}{s_{n}^{\mu_{n}}} \cdots \sum_{s_{2}=1}^{\infty} \frac{1}{s_{2}^{\mu_{2}}} \sum_{s_{1}=1}^{\infty} \frac{1}{s_{1}^{\mu_{1}}}=\left.\left.\left.\Delta_{h_{n}}^{-1} t_{n}^{\mu_{n}-1} \sum_{s_{n}=1}^{\infty} e^{-s_{n} t_{n}}\right|_{0} ^{\infty} \cdots \Delta_{h_{2}}^{-1} t_{2}^{\mu_{2}-1} \sum_{s_{2}=1}^{\infty} e^{-s_{2} t_{2}}\right|_{0} ^{\infty} \Delta_{h_{1}}^{-1} t_{1}^{\mu_{1}-1} \sum_{s_{1}=1}^{\infty} e^{-s_{1} t_{1}}\right|_{0} ^{\infty}, \\
\prod_{j=1}^{n} \Gamma\left(\mu_{j}\right) \zeta\left(\mu_{n}\right) \cdots \zeta\left(\mu_{2}\right) \zeta\left(\mu_{1}\right)=\left.\left.\left.\Delta_{h_{n}}^{-1} t_{n}^{\mu_{n}-1} \frac{1}{\left(e^{\left.t_{n}-1\right)}\right.}\right|_{0} ^{\infty} \cdots \Delta_{h_{2}}^{-1} t_{2}^{\mu_{2}-1} \frac{1}{\left(e^{\left.t_{2}-1\right)}\right.}\right|_{0} ^{\infty} \Delta_{h_{1}}^{-1} t_{1}^{\mu_{1}-1} \frac{1}{\left(e^{t_{1}}-1\right)}\right|_{0} ^{\infty}, \\
\zeta\left(\mu_{n}\right) \cdots \zeta\left(\mu_{2}\right) \zeta\left(\mu_{1}\right)=\left.\left.\left.\frac{1}{\Gamma\left(\mu_{n}\right)} \Delta_{h_{n}}^{-1} \frac{t_{n}^{\mu_{n}-1}}{\left(e^{t_{n}}-1\right)}\right|_{0} ^{\infty} \cdots \frac{1}{\Gamma\left(\mu_{2}\right)} \Delta_{h_{2}}^{-1} \frac{t_{2}^{\mu_{2}-1}}{\left(e^{t_{2}}-1\right)}\right|_{0} ^{\infty} \frac{1}{\Gamma\left(\mu_{1}\right)} \Delta_{h_{1}}^{-1} \frac{t_{1}^{\mu_{1}-1}}{\left(e^{t_{1}}-1\right)}\right|_{0} ^{\infty},
\end{gathered}
$$

which is the product of $n^{\text {th }}$-kind Riemann zeta function in the discrete case.

\subsection{One-Dimensional Laplace Transform on the Fractional} Difference Equation. Let $u(t)$ and $v(t)$ be the two functions. The Leibniz rule of noninteger order is $\stackrel{v}{\Delta}[u(t) v(t)]=\sum_{r=0}^{\infty}\left(\begin{array}{c}v \\ r\end{array}\right)^{\nu-r} \Delta u(t) \stackrel{r}{\Delta} v(t+v-r) . \quad$ Here, we present the product formula on the fractional difference operator $\quad$ as $\quad \Delta_{h}^{\nu}[u(t) v(t)]=\sum_{r=0}^{\infty}\left(\begin{array}{c}\nu \\ r\end{array}\right) \Delta_{h}^{\nu-r} u(t) \Delta_{h}^{r} v(t+$ $(\nu-r) h)$.

The following theorem plays an important role in solving the fractional difference equation by one-dimensional Laplace transform.

Theorem 8. Let $u\left(t_{1}\right)$ be a real-valued function and $s_{1}, h_{1}, v_{1}>0$. Then, we have

$$
L_{h_{1}}\left[\Delta_{h_{1}}^{v} u(t)\right]=\frac{\left(1-e^{-s_{1}^{1 / \nu_{1}} h_{1}}\right)^{\nu_{1}}}{h_{1}^{\nu_{1}}} L_{h_{1}}\left[u\left(t_{1}+v_{1} h_{1}\right)\right]-\sum_{r_{1}=1}^{\infty} \frac{\left(1-e^{-s_{1}^{1 / \nu_{1}} h_{1}}\right)^{\nu_{1}-r_{1}}}{h_{1}^{\nu_{1}-r_{1}}} \Delta_{h_{1}}^{r_{1}-1} u\left(\left(v_{1}-r_{1}\right) h_{1}\right) .
$$

Proof. Taking $u\left(t_{1}\right)=\Delta u\left(t_{1}\right)$ in (16), we get $L_{h_{1}}\left[\Delta u\left(t_{h_{1}}\right)\right]=\left.\Delta_{h_{1}}^{-1}\left[\Delta u\left(t_{h_{1}}\right) e^{-s_{1}^{h_{1} p_{1}}} t_{1}\right]\right|_{0} ^{\infty}$. Now, applying (3) and solving, we get

$$
L_{h_{1}}\left[\frac{\Delta}{h_{1}} u\left(t_{1}\right)\right]=\frac{\left(1-e^{-s_{1}^{1 / \nu_{1}} h_{1}}\right)}{h_{1}} L_{h_{1}}\left[u\left(t_{1}+h_{1}\right)\right]-u(0) .
$$

Again taking $u\left(t_{1}\right)=\Delta_{h_{1}}^{2} u\left(t_{1}\right)$, using (3) and (16), and applying (34) give

$$
L_{h_{1}}\left[\Delta_{h_{1}}^{2} u\left(t_{1}\right)\right]=\frac{\left(1-e^{-s_{1}^{1 / \nu_{1}} h_{1}}\right)^{2}}{h_{1}^{2}} L_{h_{1}}\left[u\left(t_{1}+2 h_{1}\right)\right]-\frac{\left(1-e^{-s_{1}^{1 / \nu_{1}} h_{1}}\right)}{h_{1}} u\left(h_{1}\right)-\underset{h_{1}}{ } u(0) .
$$

Continuing this process for integer $n$, we arrive at

$$
L_{h_{1}}\left[\Delta_{h_{1}}^{n} u\left(t_{1}\right)\right]=\frac{\left(1-e^{-s_{1}^{1 / \nu_{1}} h_{1}}\right)^{n}}{h_{1}^{n}} L_{h_{1}}\left[u\left(t_{1}+n h_{1}\right)\right]-\sum_{r_{1}=1}^{n} \frac{\left(1-e^{-s_{1}^{1 / \nu_{1}} h_{1}}\right)^{n-r_{1}}}{h_{1}^{n-r_{1}}} \Delta_{h_{1}}^{r_{1}-1} u\left(\left(n-r_{1}\right) h_{1}\right) .
$$


Since the order is a fraction, we consider (36) for fraction $\nu$ as mentioned in (33).

\section{4. n-Dimensional Inverse Laplace Transform}

The $n$-dimensional inverse Laplace transform is defined by $\mathscr{L}_{n(h)}^{-1}\left[U_{n}(\bar{s})\right]=u(\bar{t})=\left.\Delta \frac{-1}{n(h)} U_{n}(\bar{s}) e^{\sum_{j=1}^{n} s_{j}^{1 / v_{j}} t_{j}}\right|_{c_{j}-i \infty} ^{c_{j}+i \infty}, \quad j=1,2, \ldots, n$.
Since we can easily represent the $n$-dimensional Laplace transform of the functions mentioned, we can present some results listed as follows:

$$
\begin{aligned}
& \mathscr{L}_{n(h)}^{-1}\left[\frac{\prod_{j=1}^{n} h_{j}}{\prod_{j=1}^{n}\left(e^{-s_{j}^{1 / v_{j}} h_{j}}-1\right)}\right]=1 \\
& \mathscr{L}_{n(h)}^{-1}\left[\frac{\prod_{j=1}^{n} h_{j}}{\prod_{j=1}^{n}\left(e^{-\left(s_{j}^{1 / v_{j}}-a_{j}\right) h_{j}}-1\right)}\right]=e^{\sum_{j=1}^{n} a_{j} t_{j}}, \\
& \mathscr{L}_{n(h)}^{-1}\left[\frac{\prod_{j=1}^{n} h_{j} \sum_{\bar{r} \in \mathcal{F}\left(D_{n}\right)}(-1)^{n\left(D_{n}-\bar{r}\right)} e_{-i\left(a_{\bar{r}} h_{\bar{r}}\right)} e_{s_{D_{n}-\bar{r}} h_{D_{n}-\bar{r}}}}{2^{n}\left[\prod_{j=1}^{n}\left(\cosh s_{j}^{1 / v_{j}} h_{j}-\cos a_{j} h_{j}\right)\right]}\right]=e^{i \sum_{j=1}^{n} a_{j} t_{j}}, \\
& \mathscr{L}_{n(h)}^{-1}\left[\prod_{j=1}^{n} \frac{h_{j}^{\mu_{j}+1} \mu_{j} ! e^{s_{j} / v_{j}} h_{j}}{\left(e^{-\left(s_{j}^{1 / v_{j}}-1\right)}-1\right)}\right]=\prod_{j=1}^{n}\left(t_{j}\right)_{h_{j}}^{\left(\mu_{j}\right)}, \\
& \mathscr{L}_{n(h)}^{-1}\left[\frac{\Gamma\left(\mu_{n}\right) \ldots \Gamma\left(\mu_{2}\right) \Gamma\left(\mu_{1}\right)}{s_{n}^{\mu_{n}} \ldots s_{2}^{\mu_{2}} s_{1}^{\mu_{1}}}\right]=t_{1}^{\mu_{1}-1} t_{2}^{\mu_{2}-1} \ldots t_{n}^{\mu_{n}-1} .
\end{aligned}
$$

\section{Results and Discussion}

When $n=2, v_{1}=v_{2}=1$, and $h_{1}, h_{2} \longrightarrow 0$, in all the above results, we have
(i) $\mathscr{L}_{2}[1]=1 / s_{1} s_{2}$.
(ii) $\mathscr{L}_{2}\left[e^{a_{1} t_{1}+a_{2} t_{2}}\right]=1 /\left(s_{1}-a_{1}\right)\left(s_{2}-a_{2}\right)$.
(iii) $\mathscr{L}_{2}\left[\sin \left(a_{1} t_{1}+a_{2} t_{2}\right)\right]=a_{1} a_{2} /\left(s_{1}^{2}+a_{1}^{2}\right)\left(s_{2}^{2}+a_{2}^{2}\right)$.
(iv) $\mathscr{L}_{2}\left[\cos \left(a_{1} t_{1}+a_{2} t_{2}\right)\right]=s_{1} s_{2} /\left(s_{1}^{2}+a_{1}^{2}\right)\left(s_{2}^{2}+a_{2}^{2}\right)$.

Similarly, the following result for the hyperbolic functions can be obtained:

$$
\text { (i) } \mathscr{L}_{2}\left[t_{1}^{\mu_{1}} t_{2}^{\mu_{2}}\right]=\mu_{1} ! \mu_{2} ! / s_{1}^{\mu_{1}+1} s_{2}^{\mu_{2}+1} \text {. }
$$

These results match the formulas of the double Laplace transform of functions available in the literature.

\section{Conclusion}

The fractional frequency is used to derive the $n$-dimensional Laplace transform with shift values $h_{j}, j=1,2, \ldots, n$, that presents more accuracy outputs of the input functions such as exponential, polynomial factorial, polynomial, and trigonometric functions. Also, the numerical results and the solutions are analyzed graphically by MATLAB. The major application of this research work is also provided by considering the classical Laplace transform according to particular values of $n$ which are $v_{j}=1$ and $h_{j} \longrightarrow 0, j=1,2, \ldots, n$.

\section{Data Availability}

The data used to support the findings of this study are available from the corresponding author upon request.

\section{Conflicts of Interest}

The authors declare that they have no conflicts of interest.

\section{Acknowledgments}

This research was catalyzed and financially supported by the Tamil Nadu State Council for Science and Technology, 
Department of Higher Education, Government of Tamil Nadu.

\section{References}

[1] I. Podlubny, Fractional Differential Equations, Academic Press, San Diego, CA, USA, 1999.

[2] G. Samko, A. A. Kilbas, and S. Marichev, Fractional Integrals and Derivatives: Theory and Applications, Gordon \& Breach, Yverdon, Switzerland, 1993.

[3] A. A. Kilbas, M. H. Srivastava, and J. J. Trujillo, "Theory and application of fractional differential equations," North-Holland Mathematics Studies, Vol. 204, Elsevier, Amsterdam, Netherland, 2006.

[4] R. L. Magin, Fractional Calculus in Bioengineering, Begell House Publishers, Danbury, CT, USA, 2006.

[5] T. Abdeljawad, "On Riemann and Caputo fractional differences," Computers \& Mathematics with Applications, vol. 62, no. 3, pp. 1602-1611, 2011.

[6] F. M. Atic1 and P. W. Eloe, "A transform method in discrete fractional calculus," International Journal Difference Equations, vol. 2, no. 2, pp. 165-176, 2007.

[7] F. M. Atıcı and P. W. Eloe, "Initial value problems in discrete fractional calculus," Proceedings of the American Mathematical Society, vol. 137, no. 3, pp. 981-989, 2009.

[8] F. M. Atıcı and P. W. Eloe, "Discrete fractional calculus with the nabla operator," Electronic Journal of Qualitative Theory of Differential Equations, vol. 3, 2009.

[9] F. M. Atıcı and S. Șengül, "Modelling with fractional difference equations," Journal of Mathematical Analysis and Applications, vol. 369, no. 1, pp. 1-9, 2010.

[10] K. S. Miller and B. Ross, "Fractional difference calculus," in Proceedings of the International Symposium on Univalent Functions, Fractional Calculus and Their Applications, pp. 139-152, Nihon, Japan, 1989.

[11] T. Abdeljawad and D. Baleanu, "Fractional differences and integration by parts," Journal of Computational Analysis and Applications, vol. 13, no. 3, pp. 574-582, 2011.

[12] N. R. O. Bastos, R. A. C. Ferreira, and D. F. M. Torres, "Discrete-time fractional variational problems," Signal Processing, vol. 91, no. 3, pp. 513-524, 2011.

[13] H. L. Gray and N. F. Zhang, "On a new definition of the fractional difference," Mathematics of Computation, vol. 50, no. 182 , p. $513,1988$.

[14] T. Abdeljawad and F. M. Atici, "On the definitions of nabla fractional operators," Abstract and Applied Analysis, vol. 2012, pp. 1-13, Article ID 406757, 2012.

[15] T. Abdeljawad, F. Jarad, and D. Baleanu, "A semigroup-like property for discrete Mittag-Leffler functions," Advances in Difference Equations, vol. 2012, no. 1, p. 72, 2012.

[16] G.-C. Wu, D. Baleanu, S.-D. Zeng, and Z.-G. Deng, "Discrete fractional diffusion equation," Nonlinear Dynamics, vol. 80, no. 1-2, pp. 281-286, 2015.

[17] G.-C. Wu, D. Baleanu, and S.-D. Zeng, "Several fractional differences and their applications to discrete maps," Journal of Applied Nonlinear Dynamics, vol. 4, no. 4, pp. 339-348, 2015.

[18] S. W. Smith, The Scientist and Engineer's Guide to Digital Signal Processing, California Technical Publishing, San Diego, CA, USA, 2nd edition, 1999.

[19] M. Bohner and A. Peterson, Dynamic Equation on Time Scales: An Introduction with Applications, Birkhuser, Boston, MA, USA, 2001.
[20] A. Ivic, "Some applications of Laplace transforms in analytic number theory," Novi Sad Journal of Mathematics, vol. 45, no. 1, pp. 31-44, 2015.

[21] A. Ivic, "The Laplace transform of the fourth moment of of the zeta-function," Univ. Beograd. Publ. Elektrotehn. Fak. Ser. Mat.vol. 11, pp. 41-48, 2000.

[22] A. M. Sedletskii, Fourier Transforms and Approximations, Gordon and Breach Science Publishers, Amsterdam, Netherland, 2000.

[23] M. Jutila, “The Mellin transform of the square of Riemann's zeta-function," Periodica Mathematica Hungarica, vol. 42, pp. 179-190, 2001.

[24] I. A. Abbas and M. Marin, "Analytical solution of thermoelastic interaction in a half-space by pulsed laser heating," Physica E: Low-Dimensional Systems and Nanostructures, vol. 87, pp. 254-260, 2017.

[25] M. Marin, M. A. Othman, and I. Abbas, "An extension of the domain of influence theorem for generalized thermoelasticity of anisotropic material with voids," Journal of Computational and Theoretical Nanoscience, vol. 12, no. 8, pp. 1594-1598, 2015.

[26] M. Marin, I. Abbas, and R. Kumar, "Relaxed Saint-Venant principle for thermoelastic micropolar diffusion," Structural Engineering and Mechanics, vol. 51, no. 4, pp. 651-662, 2014.

[27] M. I. A. Othman and I. A. Abbas, "Effect of rotation on plane waves at the free surface of a fibre-reinforced thermoelastic half-space using the finite element method," Meccanica, vol. 46, no. 2, pp. 413-421, 2011.

[28] G. Britto Antony Xavier, B. Govindan, S. John Borg, and M. Meganathan, "Generalized Laplace transform arrived from an inverse difference operator," Global Journal of Pure and Applied Mathematics, vol. 12, no. 3, pp. 661-666, 2016.

[29] L. Debnath, "The double Laplace transforms and their properties with applications to functional, integral and partial differential equations," International Journal of Applied and Computational Mathematics, vol. 2, no. 2, pp. 223-241, 2016.

[30] M. Meganathan, G. Britto Antony Xavier, and T. Abdeljawad, "Modeling with fractional Laplace transform by h-difference operator," Progress in Fractional Differentiation and Applications, In press.

[31] S. Pinelas, M. Meganathan, M. Murugesan, and B. A. X. G. Gnanaprakasam, "Fractional frequency Laplace transform by inverse difference operator with shift value," Open Journal of Mathematical Sciences, vol. 3(2019), no. 1, pp. 121-128, 2019. 